\title{
Orbital-overlap model for minimal work functions of cesiated metal surfaces
}

\author{
Sharon H. Chou ${ }^{* 1}$, Johannes Voss* ${ }^{2,3}$, Igor Bargatin ${ }^{1,4}$, Aleksandra Vojvodic ${ }^{2,3}$, \\ Roger T. Howe ${ }^{1}$, and Frank Abild-Pedersen ${ }^{3}$ \\ ${ }^{I}$ Department of Electrical Engineering, Stanford University, Stanford, California 94035, USA \\ ${ }^{2}$ Department of Chemical Engineering, Stanford University, Stanford, California 94035, USA \\ ${ }^{3}$ SUNCAT Center for Interface Science and Catalysis, \\ SLAC National Accelerator Laboratory, Menlo Park, California 94205, USA \\ ${ }^{4}$ Department of Mechanical Engineering and Applied Mechanics, University of Pennsylvania, Philadelphia, Pennsylvania 19104, \\ USA
}

\begin{abstract}
We introduce a model for the effect of cesium adsorbates on the work function of transition metal surfaces. The model builds on the classical point-dipole equation by adding exponential terms that characterize the degree of orbital overlap between the $6 s$ states of neighboring cesium adsorbates and its effect on the strength and orientation of electric dipoles along the adsorbate-substrate interface. The new model improves upon earlier models in terms of agreement with the work function-coverage curves obtained via first-principles calculations based on density functional theory (DFT). All the cesiated metal surfaces have optimal coverages between 0.6 to 0.8 monolayers (ML), in accordance with experimental data. All the cesiated tungsten surfaces we have considered are shown to have minimum work functions (MWF) lower than most cesiated metals, also in accordance with experiments.
\end{abstract}

PACS: $73.30 .+\mathrm{y}, 68.43 . \mathrm{Bc}, 73.20 . \mathrm{Hb}$

\section{INTRODUCTION}

The work function is one of the most fundamental properties of a metallic surface, important in determining the material's applicability as a contact electrode ${ }^{1,2}$ or electron emitter ${ }^{3}$. Surfaces with ultralow work functions could therefore improve technologies requiring precise control of contact barriers such as organic and printed electronics ${ }^{2}$ or a variety of devices based on electron emission, ranging from fluorescent light bulbs ${ }^{4,5}$ to $\mathrm{THz}$ sources $^{6}$, thermionic energy converters $(\mathrm{TECs})^{7}$, and the photon-enhanced thermionic emission converters (PETEC) $^{8}$. For TEC and PETEC, in particular, discovery of thermally stable materials with work functions of less than $1 \mathrm{eV}$ would allow thermionic conversion of high-temperature $\left(>500{ }^{\circ} \mathrm{C}\right.$ ) heat or solar radiation directly to electricity with efficiencies exceeding $50 \%$.

Among elemental materials, alkali metals are known to have the lowest work functions, with cesium having the lowest at $\approx 2.0 \mathrm{eV}^{9}$. However, in practical applications, a number of alloys and compounds, such as thoriated tungsten ${ }^{10,11,12,13}$ and lanthanum hexaboride ${ }^{14,15}$, are used because they offer relatively low work functions $(\approx 2.5 \mathrm{eV})$ in combination with much better chemical and thermal stabilities. It has also been known since the $1930 \mathrm{~s}^{16,17}$ that it is possible to create surfaces with work functions lower than those of any elemental, bulk materials by using coatings with thicknesses on the order of a monolayer. For example, the most popular cesiated metal surface - polycrystalline tungsten with a submonolayer coating of cesium (W/Cs system) - has a minimum work function (MWF) of $\approx 1.5 \mathrm{eV}$, which is significantly lower than the work functions of both cesium $(\approx 2.0 \mathrm{eV})$ and tungsten $(\approx 4.5 \mathrm{eV})$ themselves ${ }^{18}$. In all such cases, the work function of the substrate is lowered due to partial transfer of electron charge from the adsorbate to the substrate and the resulting formation of surface dipoles.

In this study, we focus on cesium as the adsorbate because it generally induces the largest decrease in the work function among atomic adsorbates ${ }^{19,20,21,22}$. As a starting point for designing new materials, we perform a first-principles-based screening study of trends in minimal work functions that can be obtained using cesiated $3 d-, 4 d$ - and $5 d$-transition metals. Based on the observed results, we introduce a new model of dipole-induced work function lowering that significantly improves the agreement with the simulated results and experiments. The new model takes into account not only electrostatic interactions but also covalent interactions due to orbital overlap among neighboring adsorbates.

The article is structured as follows: In Section II below, we briefly describe the methods of DFT calculations. In Section III, we explain the general dependence of the work function on cesium coverage in the low, intermediate, and high coverage regimes. In Section IV, we describe the classic point-dipole 
model of work function reduction due to atomic adsorbates and discuss its limitations. In Section V, we introduce the new orbital-overlap model. Finally, in Section VI we present the results of DFT simulations, discuss how well they agree with various dipole models, and determine the lowest work functions attainable with different transition metal substrates.

\section{METHODS}

We study work functions of cesiated transition metal substrates using first-principles calculations within the framework of density functional theory (DFT) ${ }^{23}$. The ion cores are described by ultrasoft pseudopotentials ${ }^{24}$, as implemented in the plane-wave code Dacapo ${ }^{25}$. For the exchange-correlation functional, the Revised Perdew-Burke-Ernzerhof $(\mathrm{RPBE})^{26}$ version of the generalized gradient approximation is employed. For the expansion of the Kohn-Sham ${ }^{27}$ bands a kinetic energy cutoff of $340 \mathrm{eV}$ is used, and Brillouin zone sampling is performed with a grid spacing of at most $0.05 \AA^{-1}$.

All calculations are carried out without spin polarization. We find that the difference in the work functions predicted by magnetic and non-magnetic models is only on the order of $50 \mathrm{meV}$ due to pinning of the Fermi level with respect to the vacuum electrostatic potential by the Cs adsorbates.

Using relaxed bulk lattice constants, supercell calculations are performed with $20 \AA$ of vacuum between the slabs. We find that a slab consisting of a minimum of four metal layers, where the two topmost layers are allowed to relax, is required for the work function to converge. A dipole correction is applied to compensate for the symmetry-breaking with respect to the fixed and free surface of the slab, reducing the number of layers needed compared to a full relaxation of the entire $\operatorname{slab}^{28}$.

\section{DEPENDENCE OF WORK FUNCTION ON ADSORBATE COVERAGE}

Figure 1 shows the typical dependence of the work function on the cesium coverage as observed both in experiments $^{20,29}$ and DFT simulations ${ }^{19,30}$. Initially, the work function decreases approximately linearly with increasing coverage as the dipoles associated with individual adsorbate atoms practically do not interact with each other. However, as the coverage increases and the average distance between the adsorbate cesium atoms decreases, the dipoles begin to interact, at first electrostatically as point dipoles and then covalently via the extended electron clouds of cesium $^{31}$. As a result of these interactions, the dipole associated with each individual adsorbate atom decreases (i.e., depolarization occurs), causing the work function to reach a minimum and then increase again, eventually saturating at the value corresponding to the work function of bulk cesium.

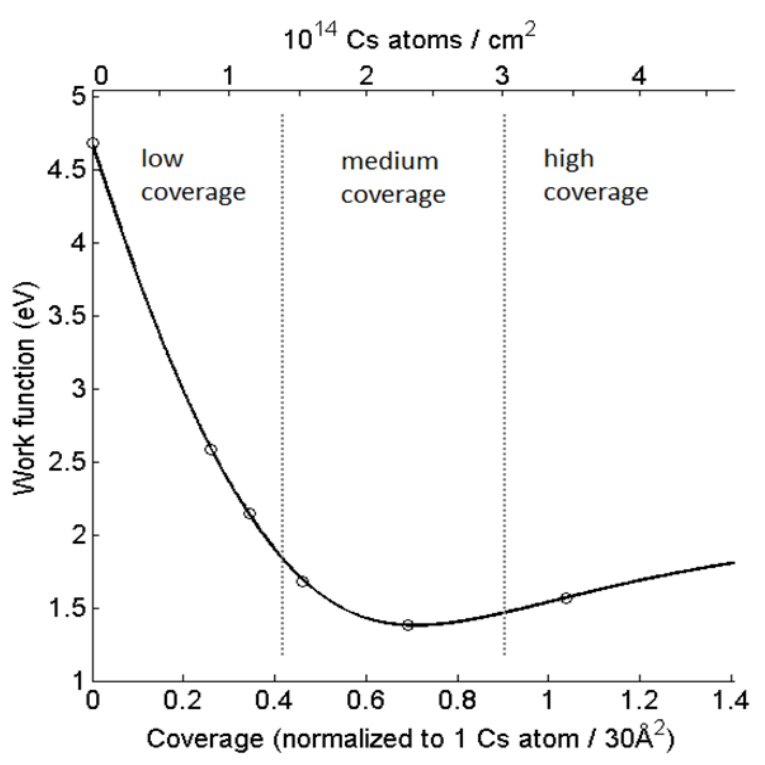

FIG. 1. DFT-calculated work function vs. Cs coverage on the $\mathrm{W}(110)$ surface. The work function is in units of $\mathrm{eV}$ and the coverage is normalized to $1 \mathrm{Cs}$ atom $/ 30 \AA^{2}$, the unit surface area of $\mathrm{Cs}(110)$ relaxed within DFT. Vertical dotted lines indicate the regimes of low coverage, medium coverage (where the work function reaches a minimum), and high coverage (where the work function approaches that of the bulk cesium).

We can qualitatively characterize the three regimes of the adsorbate-induced work function reduction in Fig. 1 as follows: At low coverage (Fig. 2a), the distance between neighboring adsorbate atoms is so large that they can be treated as point dipoles, and simple electrostatic interactions between the point dipoles provide an adequate description. Conversely, at high coverage (Fig. 2c), strong covalent interactions dominate and the dipole can only be adequately modeled quantum mechanically. However, there is an intermediate regime of crossover between these classical and quantum-mechanical contributions. It is in this intermediate region of relatively weak covalent bonds (Fig. 2b) that the work function typically reaches a minimum. Therefore, it is particularly important to have a model that adequately describes the dependence of the work 
function on the adsorbate coverage in this intermediate regime.
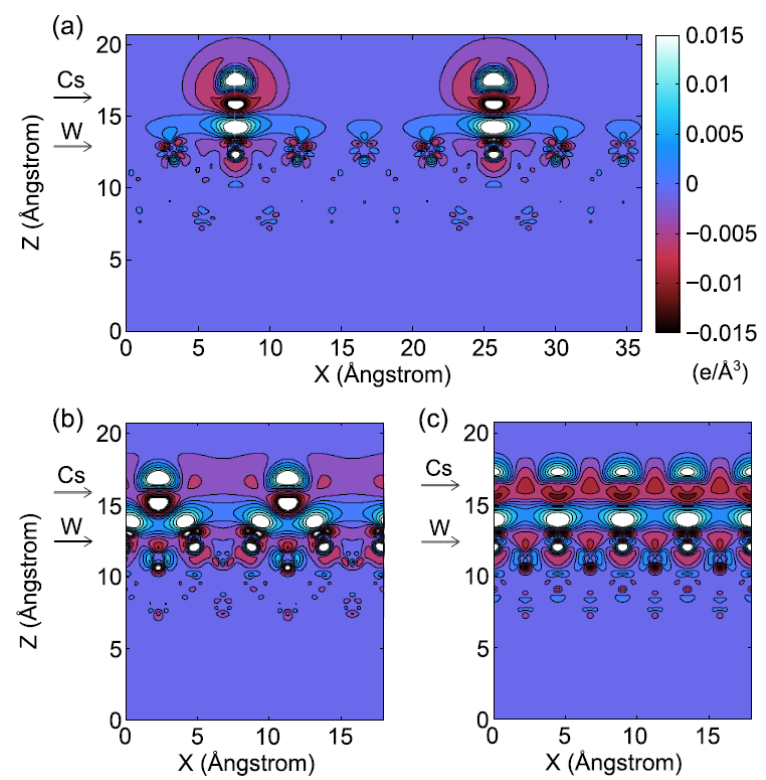

FIG. 2. (Color online) $X-Z$ spatial profiles of net valence electron charge density showing $\mathrm{Cs}$ adsorbed on the $\mathrm{W}(100)$ surface at (a) low, (b) near optimal, and (c) high coverage. The profiles show isocontours of the change in electron charge density between a bare and cesiated tungsten surface. Cyan blue (light) = electron-rich, red $($ dark $)=$ electron-poor. The centers of the Cs atoms and the top layer of the $\mathrm{W}$ atoms are marked by arrows.

\section{CLASSICAL POINT-DIPOLE MODEL}

At sufficiently low coverage, a single alkali atom is predicted to be adsorbed as an ion ${ }^{30,32,33}$, with the corresponding dipole formed normal to the surface (e.g., Fig. 2a). There are two competing effects as the number of adsorbates increases: the increase of the overall dipole density and the depolarization due to electrostatic interaction between the dipoles. These two effects are accounted for in the simple classical dipole model through the depolarization equation ${ }^{22}$ :

$$
\Delta \Phi=\frac{-1}{\varepsilon_{0}} \frac{e N \mu_{0_{z}}}{1+\left(c \alpha N^{3 / 2}\right) /\left(4 \pi \varepsilon_{0}\right)}=\frac{b_{1} N}{1+b_{2} N^{3 / 2}},
$$

where $\Delta \Phi$ is the reduction in the work function, $N$ is the number of surface-coating adsorbates per unit area, $\mu_{0_{z}}$ is the surface-normal dipole moment associated with each adsorbate in the limit of very low surface coverage (i.e., without depolarization), $\alpha$ is the linear polarizability of the surface dipoles, and $c(\approx 9)$ is a dimensionless parameter that weakly depends on the exact spatial arrangement of the dipoles on the surface. This model is valid at relatively low surface coverage where the dipole strength responds linearly to local fields, i.e., where the dipoles do not rotate or start interacting through covalent bonding among adsorbate atoms ${ }^{22}$. Since the initial dipole moment $\mu_{0_{z}}$ is generally governed by the difference between the electronegativities of the adsorbate and the substrate, the initial slope of Eq. (1) is particularly large for the combination of cesium and highly electronegative transition metals such as tungsten.

In the intermediate regime, however, the adsorbatesubstrate bond adopts a partially covalent character as purely covalent bonds start to form between the adsorbates themselves ${ }^{30,31,33}$ (Fig. 3). At the optimal cesium coverage corresponding to the minimum work function (MWF), there is significant orbital overlap between neighboring cesium atoms (Fig. 2b). In the regime of very high surface coverage (Fig. 2c), adsorbates form a layer that is essentially covalent $^{31,33}$, and the work function of bulk cesium is reached (Fig. 1).

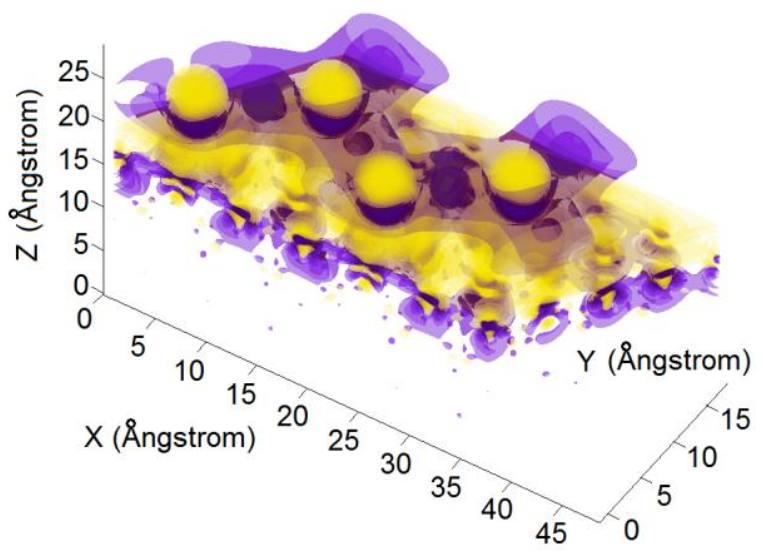

FIG. 3. (Color online) Net valence electron charge distribution of Cs atoms adsorbed on the W(110) surface at near-optimal coverage (i.e., where the work function is at its minimum). The figure shows isosurfaces of the change in electron charge density between a bare and cesiated tungsten surface. The isosurfaces correspond to charge density spaced by approximately $\approx 1.5 \times 10^{-3} \mathrm{e} / \AA^{3}$. Violet (dark gray) indicates net positive charge (electron-poor), while yellow (light gray) indicates net negative charge (electron-rich). The maximum change in the positive charge density is $\approx 5.17 \times 10^{-2} \mathrm{e} / \AA^{3}$ and the maximum change in the negative charge density is $\approx 4.04 \times 10^{-2} \mathrm{e} / \AA^{3}$.

To account for the onset of orbital overlap, which is crucial for an accurate description of the work function behavior around its minimum, a model 
beyond the classical electrostatic description according to Eq. (1) is needed.

\section{ORBITAL-OVERLAP MODEL}

Due to the large extent of the Cs $6 s$ states, overlap becomes important at coverages as low as $\approx 2 \times 10^{14}$ atoms $/ \mathrm{cm}^{2}(\approx 0.5 \mathrm{ML})$. On one hand, the large extent of the $6 s$ states renders the assumption of electrostatically interacting point-dipoles in Eq. (1) invalid. On the other hand, the formation of Cs-Cs bonds introduces further depolarization as charge transfer to these covalent bonds weakens the dipoles normal to the surface.

We will model these two effects by introducing additional overlap terms into the classical model described by Eq. (1):

$$
\Delta \Phi=\frac{c_{1} N}{1+c_{2} N^{3 / 2}\left(1-\mathrm{e}^{-c_{3} / N}\right)+c_{4} \mathrm{e}^{-c_{3} / N}}
$$

in which the exponential terms $\exp \left(-c_{3} / N\right)$ describe the orbital overlap between neighboring adsorbates, $c_{3}$ is inversely proportional to the areal spread of the $6 s$ Gaussian states, and $c_{4}$ describes the strength of depolarization due to orbital overlap. The $1 / N$ dependence in the exponent of the overlap terms stems from the distance dependence of the integral of two overlapping Gaussians. Eq. (2) is designed to describe the onset of overlap-induced depolarization between neighboring $\mathrm{Cs}$ sites and hence the intermediate coverage regime.

The factor $\left[1-\exp \left(-c_{3} / N\right)\right]$ is selected as the simplest form to scale down the diverging classical depolarization term. The additional depolarization term $c_{4} \exp \left(-c_{3} / N\right)$ accounts for the charge density transfer from the dipoles normal to the surface to the in-plane orbitals that covalently bind the Cs adsorbates. Further terms could be introduced if a description of the saturation in the high coverage regime was required. However, we do not consider them here, since we are primarily interested in the MWF region, which generally occurs at the crossover from classical point-dipole to overlap-dominated behavior.

When comparing the predictions of Eq. (2) to the results of DFT simulations, it is important to keep the
Cs distances in the in-plane directions approximately equal, making triangular or square lattices ideal. A poorly chosen uneven spacing of adsorbates would lead to an increase of the total energy and is therefore not representative of thermodynamically stable configurations. In addition, it can significantly increase the depolarization compared to the case of the same coverage with an optimally arranged Cs adsorbate lattice. The requirement of evenly spaced adsorbates constrains the number of coverages that can be studied using small supercells, including systems with more than one adsorbate per cell. Excluding the work function at zero coverage, which corresponds to a parameter-independent limit of Eq. (2), we typically calculated six DFT data points per transition metal substrate and surface orientation. There are, however, strong correlations between the four fitting parameters - the correlations lie between $50 \%$ and $90 \%$, with particularly large values for the correlations between $c_{1}$ and $c_{2}$, as well as $c_{3}$ and $c_{4}$, respectively. This result indicates that an even smaller number of fitting parameters could be sufficient if one chose a different functional form of Eq. (2).

\section{RESULTS AND DISCUSSION}

We examine the close-packed surfaces $\mathrm{HCP}(0001)$, FCC(111), and BCC(110), as well as the more open surfaces FCC(110) and BCC(100) of $3 d-, 4 d-$, and $5 d$-transition metals (except for Tc and $\mathrm{Mn}$ ). We consider these open surfaces since the work functions of the technologically important polycrystalline substrates correspond to an average over surfaces of different orientations. Empirically, the work functions measured for $\mathrm{FCC}(110)$ and $\mathrm{BCC}(100)$ substrates provide the best estimates for the observed values of polycrystalline samples ${ }^{18,34}$.

Despite the large span of different transition metals, the curve of the work function versus coverage has similar characteristics, as was discussed above. To illustrate the characteristic coverage dependence of the work functions, we show three representative systems of cesiated, close-packed transition metal surfaces in Fig. 4. The cesiated $\operatorname{Ag}(111)$, W(110), and $\mathrm{Pt}(111)$ surfaces have work function minima at a normalized coverage of approximately $0.5,0.7$, and 0.75 monolayer (ML), respectively.

The orbital-overlap model (Eq. 2) demonstrates dramatically better agreement with the DFT data (Fig. 4a) than either the classical dipole model (Fig. $4 \mathrm{~b})$ or the modified Gyftopoulos-Levine (G-L) 
model $^{32,35}$ (Fig. 4c). The classical dipole model exhibits reasonable fit at the low coverage regime but tends to overestimate the curve minimum and underestimate the work function in the high coverage regime. A modified G-L model was devised by Jensen et $a l .^{35}$ to incorporate empirical parameters such as covalent radii, although the fit tends to overestimate the work function (Fig. 4c). The modified G-L model also requires reconciliation of different experimental values such as atomic surface densities ${ }^{16,36,37,38}$. In addition, the nature of the polynomial fit in the modified G-L model results in nonphysical deviations at coverages above $1 \mathrm{ML}$ (Fig. 4c).

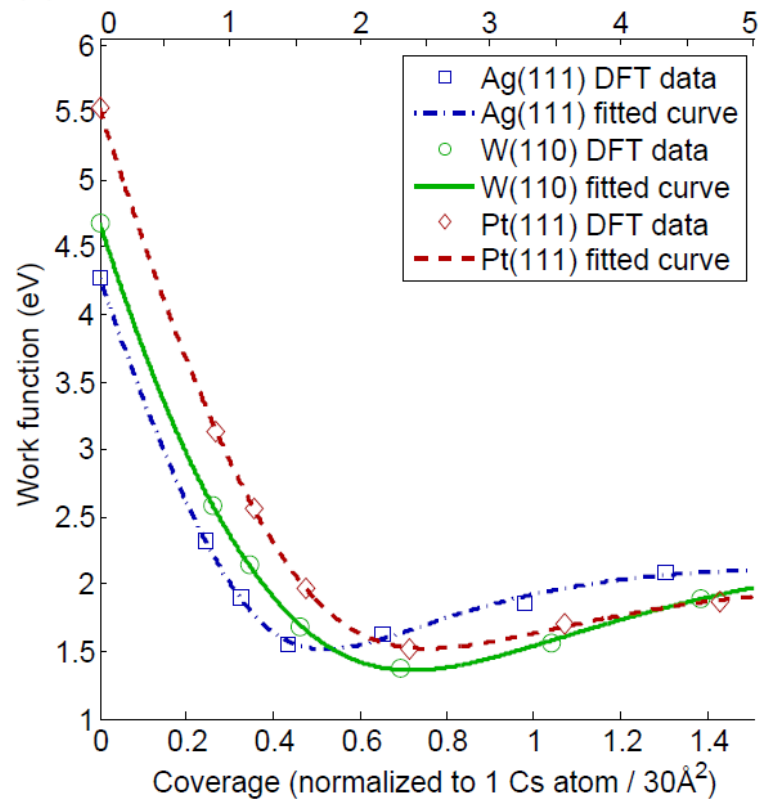

(b) $10^{14} \mathrm{Cs}$ atoms $/ \mathrm{cm}^{2}$

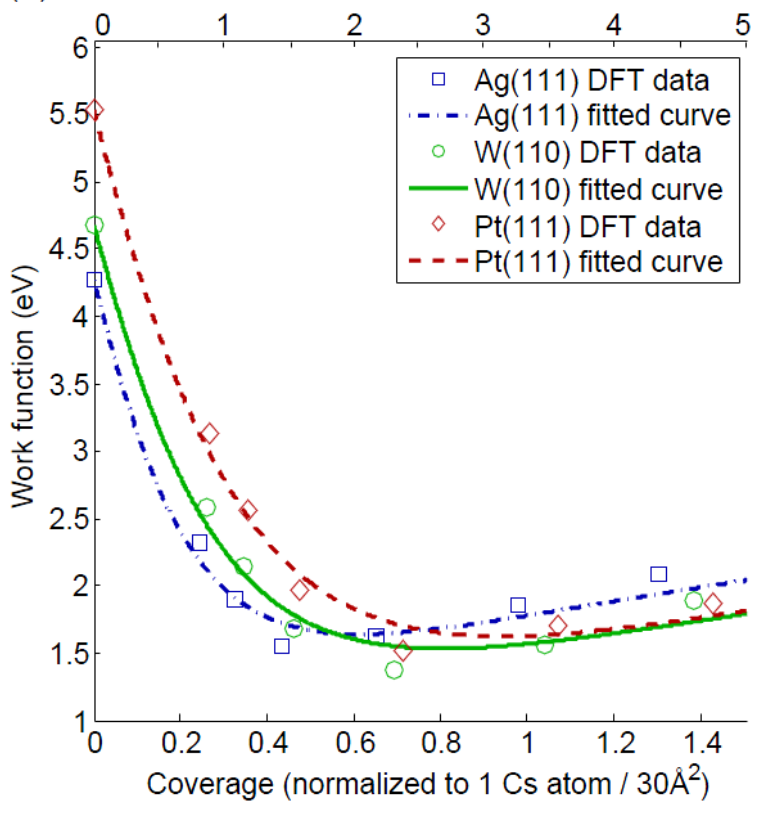

(c)

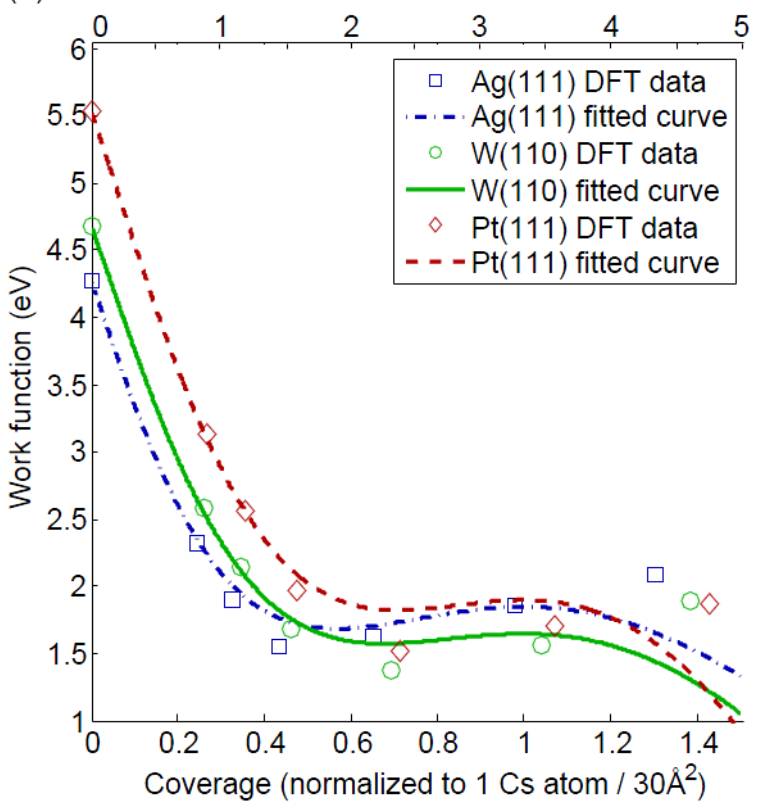

FIG. 4. Work function vs. coverage fit comparison for Csadsorbed close-packed Ag, W, and Pt surfaces. (a) Orbitaloverlap model, (b) classical dipole, and (c) modified Gyftopoulos-Levine model (Eq. 43 in Ref. 35).

Figures 5a and 5b show the calculated MWFs and optimal coverages for the metal substrates studied. In general, the cesiated transition metal surfaces have optimal coverages between 0.6 ML and 0.8 ML, in accordance with known experimental data ${ }^{20,29}$. The minimum work functions (MWFs) vary between 1.35 
and $1.8 \mathrm{eV}$, with the lowest work functions obtained for tungsten (W) and gold (Au) substrates. The lower MWF of these metal substrates are explained by their high electronegativities ( $\mathrm{W}$ at 2.36 , Au at 2.54$)^{39}$ due to a large atomic number $Z$ in the case of $\mathrm{Au}$ and half-filled outermost $s$ - and $d$-orbitals in the case of W. For tungsten, there is half-filling in both $s$ and $d$ due to $s$ - $d$ hybridization.

In experiments and for practical applications, cesiated tungsten has been much more widely-used than gold due to tungsten's refractory properties. The optimal coverages of cesium on tungsten reported in the literature range from $1.8 \times 10^{14} \mathrm{Cs}$ atoms $/ \mathrm{cm}^{2}$ (Refs. 40 and 41 ) to $3.0 \times 10^{14} \mathrm{Cs}$ atoms $/ \mathrm{cm}^{2}$ (Ref. 42) and $3.5 \times 10^{14} \mathrm{Cs}$ atoms $/ \mathrm{cm}^{2}$ (Ref. 43). These results are in agreement with our DFT-based predictions of approximately $2.3 \times 10^{14} \mathrm{Cs}$ atoms $/ \mathrm{cm}^{2}$ (Fig. 4).

It has long been known that bare (uncesiated) metal substrates generally have lower work functions for the open, i.e., less stable surfaces (e.g., BCC(100) and FCC(110)) than for the close-packed surfaces (e.g., BCC(110) and FCC(111)). This result is due to the smoothing of the surface electric charge distribution (the Smoluchowski effect) that lowers the work function ${ }^{28,44}$. Moreover, it has been found empirically that a higher substrate work function usually leads to a lower MWF upon cesium adsorption $^{45}$. Our results shown in Figs. 5a and 5b agree with both of these experimental results.

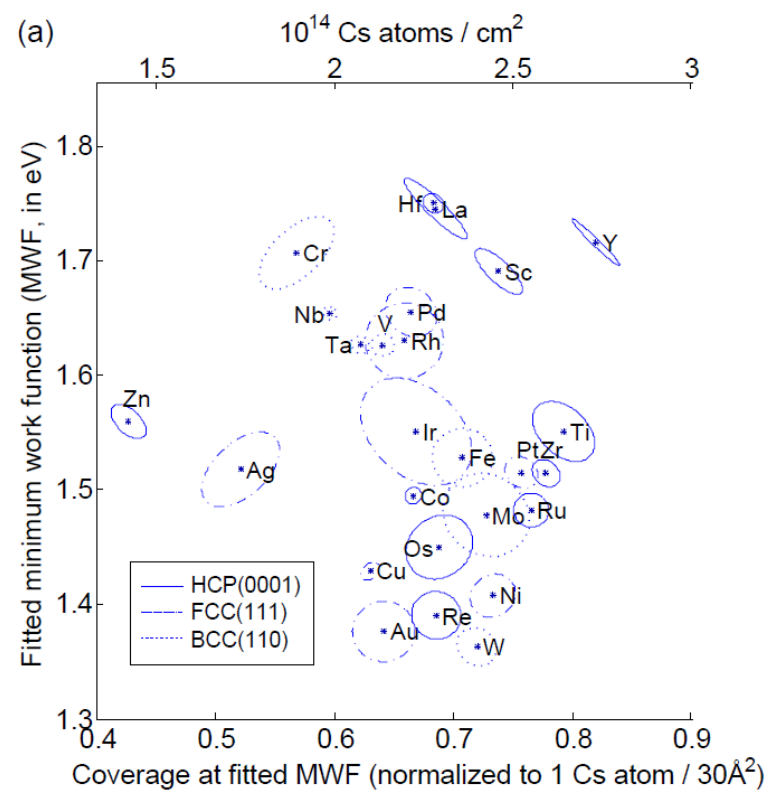

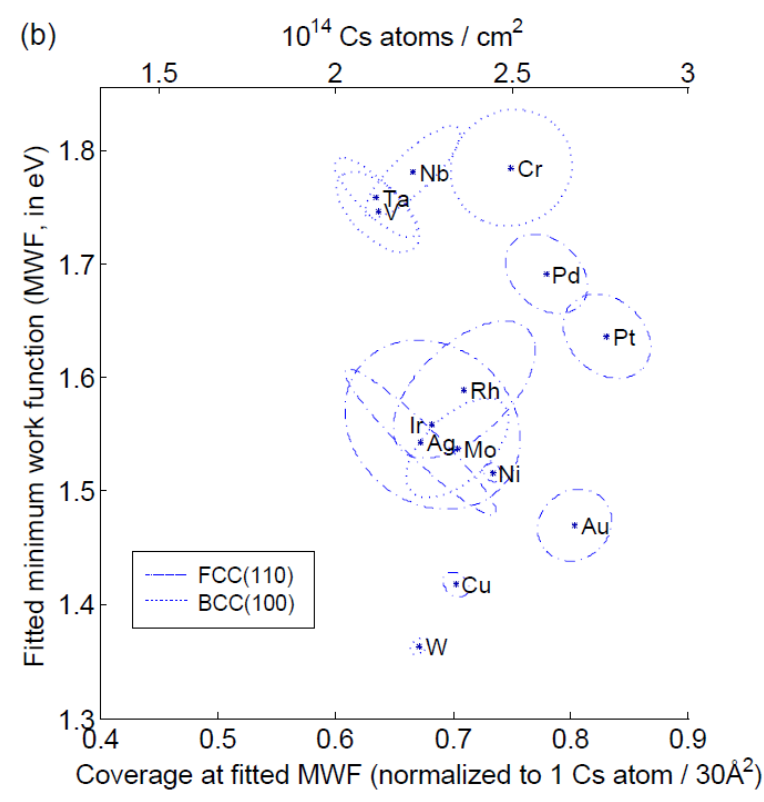

FIG. 5. Minimum work functions (MWF) and their corresponding optimal coverages as calculated by the orbital-overlap model for (a) close-packed body-centered, face-centered, and hexagonal transition metal surfaces, and (b) open, or loose-packed body-centered and face-centered metal surfaces. The elliptical outlines are 1- $\sigma$ confidence intervals while the tilting illustrates the correlation of optimal coverage and MWF based on finite-difference calculations of the fitting parameters in Eq. (2).

Some of the cesiated surfaces we studied have the same crystal symmetry and similar lattice constants but different optimal coverages. It is thus interesting to compare their charge densities (e.g., Ag vs. Au and $\mathrm{Zn}$ vs. Ru) and thereby test the assumptions of our orbital-overlap model. The binding energy of cesium on silver at intermediate to high coverage is only about half as large as in the case of cesiated gold. The weaker binding means more in-plane electron density concentration and hence more Cs-Cs orbital overlap in the case of silver than of gold (Fig. 6a). A similar comparison can be made between zinc and ruthenium (Fig. 6b). In both cases, a larger spread of Cs orbitals implies a higher overlap between them, corresponding to a lower electron density near the centers of $\mathrm{Cs}$ atoms and a higher electron density in between the neighboring $\mathrm{Cs}$ atoms (cf. Figs. 6a and $6 b)$. These differences in the degree of orbital spread and overlap lead to lower optimal coverages in Ag and $\mathrm{Zn}$ than in $\mathrm{Au}$ and $\mathrm{Ru}$, respectively, confirming that the orbital overlap terms in Eq. (2) are in fact crucial for determining optimal coverages and MWF from supercell simulations. 


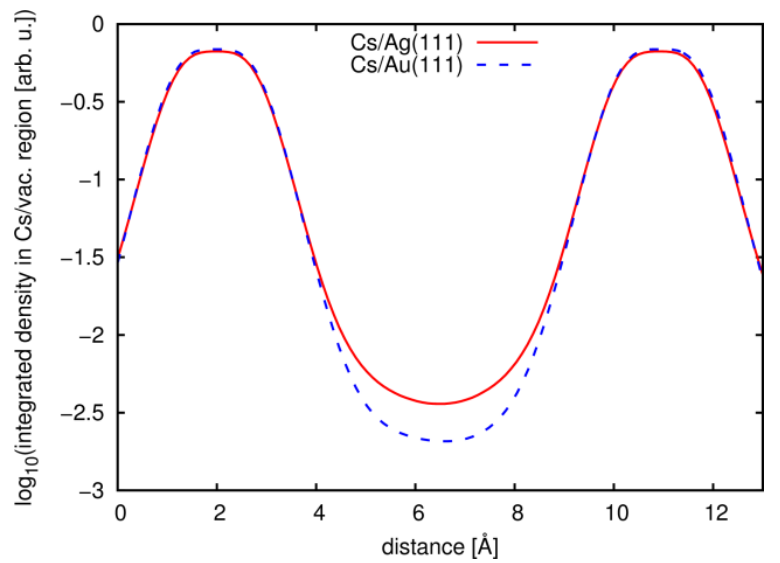

(b)

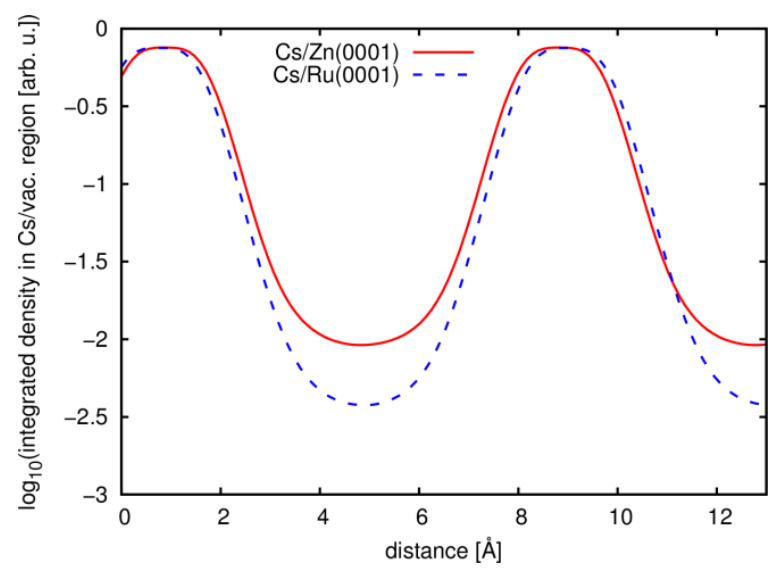

FIG. 6. Valence electron charge density plot of Cs adsorbed on (a) $\mathrm{Ag}(111)$ and $\mathrm{Au}(111)$, (b) $\mathrm{Zn}(0001)$ and $\mathrm{Ru}(0001)$ at the same coverage in $3 \times 3$ cells, integrated over one of the in-plane lattice vectors and from the Cs centers into vacuum for the distance of two ionic radii of Cs.

We note that the reason for the weaker Cs-bonding on $\mathrm{Ag}$ and $\mathrm{Zn}$ is due to lower-lying $d$-bands. When comparing the $d$-projected density of states for $\mathrm{Cu}$, $\mathrm{Ag}$ and $\mathrm{Au}$ (Fig. 7), Ag has the lowest $d$-band center and shows little overlap with the Cs-6s-projections, particularly at about 1-2 eV below the Fermi level. The $d$-band center of Au breaks the trend of lowering the $d$-band center with increasing atomic number. It is higher than that of $\mathrm{Ag}$ because of relativistic effects ${ }^{46}$. For both Ag and Zn, there are fewer $d$-states in the vicinity of the Fermi level than in the case for the other transition metals. Hence, there is less interaction with the Cs- $6 s$-states and the binding energy is lower.
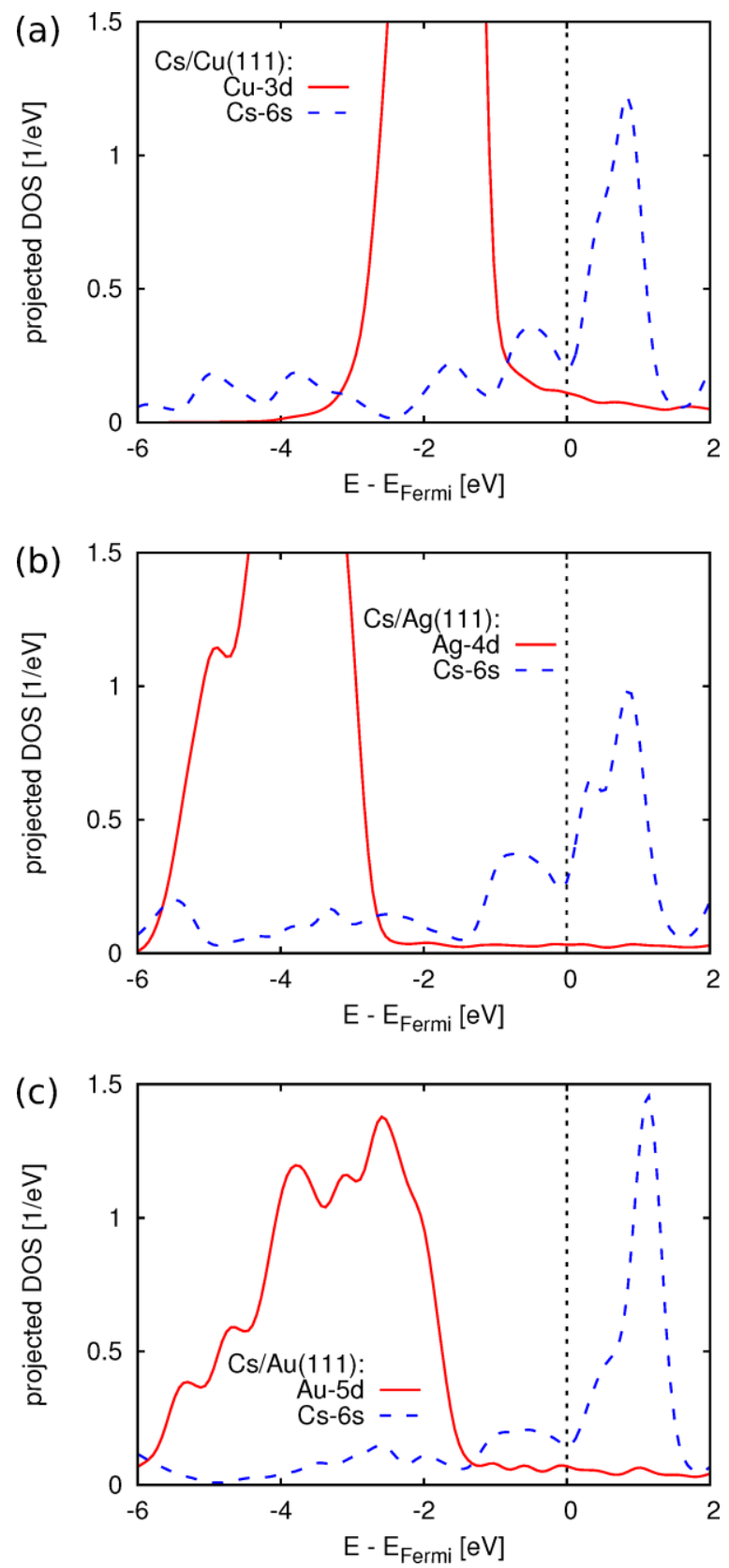

FIG. 7. $d$-projected and Cs-6s-projected density of states (DOS) for Cs adsorbed on (a) $\mathrm{Cu}(111),(b) \mathrm{Ag}(111)$ and (c) $\mathrm{Au}(111)$ with one adsorbate per $3 \times 3$ supercell. The $d$ projections are averaged over the transition metal sites in the surface layer. Lattice vectors and atomic coordinates are kept fixed at the values of $\mathrm{Au}(111)$ for all three cases (a)-(c), to exclude effects of structural relaxation (which do not lead to qualitative differences here). The DOS plots show that the differences in Cs-binding can indeed be attributed to purely electronic effects.

\section{CONCLUSION}


We have presented a new model that characterizes the effect of cesium coverage on the work function of transition metal surfaces based on the degree of orbital overlap among the cesium adsorbate atoms, in addition to electrostatic effects. We find that the inclusion of overlap terms is necessary to accurately describe the work function behavior near its minimum. The model is able to predict the optimal coverages and the trends in minimum work functions for cesiated transition metals, in general agreement with experimental data. In particular, the (100) surface of tungsten, which provides an estimate for the average work function of the widely used polycrystalline tungsten, is predicted to have the lowest work function among all transition metals.

Since the lateral dipoles are less extended for stronger Cs-bonding to the surface, engineering of surface states could lead to new materials where the onset of orbital overlap is delayed and hence, even lower work functions can be achieved. For example, a nanostructured surface with feature size on the order of $1 \mathrm{~nm}$ could be designed to contain a high density of under-coordinated sites on the surface, or have more electronic states near the Fermi level to decrease the work function.

We have shown here that the work functions of cesiated single-element transition metal surfaces cannot get lower than that of tungsten, at about 1.3 $\mathrm{eV}$. In the future, we will therefore study compound structures, e.g., interstitial elements similar to semiconductor doping, or super-lattice substrates, in order to increase the net dipole strength perpendicular to the surface and decrease the work function beyond what is possible with a simple atomic adsorbate on an elemental transition metal substrate.

\section{ACKNOWLEDGMENT}

Valuable discussions with Prof. Jens Nørskov and assistance from Dr. Chris O'Grady of the SUNCAT group are gratefully acknowledged. FAP and AV gratefully acknowledge support from the U.S. Department of Energy under contract number DEAC02-76SF00515. This work is supported by grants from the Global Climate and Energy Project (GCEP) at Stanford University and the National Science Foundation (NSF) Graduate Research Fellowship Program (GRFP) (S. H. Chou).

\section{APPENDIX}

TABLE I. Coefficients of the orbital-overlap model for $\operatorname{Ag}(111), \mathrm{W}(110)$, and $\mathrm{Pt}(111)$. Units are defined with respect to the normalized surface coverage, $N$

(Eq. 2).

\begin{tabular}{lrccc}
\hline \hline & $\begin{array}{c}c_{1} \\
\left(\mathrm{eV} \cdot \AA^{2}\right)\end{array}$ & $\begin{array}{c}c_{2} \\
\left(\AA^{-4 / 3}\right)\end{array}$ & $\begin{array}{c}c_{3} \\
\left(\AA^{-2}\right)\end{array}$ & $\begin{array}{c}c_{4} \\
(\text { dimen- } \\
\text { sionless })\end{array}$ \\
\hline $\operatorname{Ag}(111)$ & -9.3596 & 1.3742 & 2.0900 & 14.3668 \\
$\mathrm{~W}(110)$ & -9.7682 & 1.6081 & 3.4370 & 17.3524 \\
$\operatorname{Pt}(111)$ & -10.0932 & 0.9504 & 2.6771 & 10.2959 \\
\hline \hline
\end{tabular}

* Authors contributed equally to this work.

${ }^{1}$ L. Lindell, A. Burquel, F. L. E. Jakobsson, V. Lemaur, M. Berggren, R. Lazzaroni, J. Cornil, W. R. Salaneck, and X. Crispin, Chem. Mater. 18, 4246 (2006).

${ }^{2}$ Y. Zhou, C. Fuentes-Hernandez, J. Shim, J. Meyer, A. J. Giordano, H. Li, P. Winget, T. Papadopoulos, H. Cheun, J. Kim, et al., Science 336, 327 (2012).

${ }^{3}$ S. Yamamoto, Rep. Prog. Phys. 69, 181-232 (2006).

${ }^{4}$ M. Wada, S. Gotoh, and S. Kurumada, J. Plasma Fusion Res. Ser. 8, 1366 (2009).

${ }^{5}$ S. Watanabe, T. Watanabe, K. Ito, N. Miyakawa, S. Ito, H. Hosono, and S. Mikoshiba, Sci. Technol. of Adv. Mater. 12, 034410 (2011).

6 J. P. Snapp, J.-H. Lee, J Provine, I. Bargatin, R. Maboudian, T.H. Lee, and R.T. Howe, "Sidewall Silicon Carbide Emitters for Terahertz Vacuum Electronics", 2012 Solid-State Sensor and Actuator Workshop, Hilton Head Isl., SC, June 3-7 (2012).

${ }^{7}$ J.-H. Lee, I. Bargatin, J. Provine, W. Clay, J. Schwede, F. Liu, R. Maboudian, N. Melosh, Z.-X. Shen, R.T. Howe, Tech. Dig. PowerMEMS (2009).

${ }^{8}$ J. W. Schwede, I. Bargatin, D. C. Riley, B. E. Hardin, S. J. Rosenthal, Y. Sun, F. Schmitt, P. Pianetta, R. T. Howe, Z.-X. Shen, et al., Nature Mater. 9, 762 (2010).

${ }^{9}$ H. B. Michaelson, J. Appl. Phys. 48, 4729-33 (1977).

${ }^{10}$ W. B. Nottingham, Phys. Rev. 49, 78 (1936).

${ }^{11}$ A. King, Phys. Rev. 53, 570 (1938).

12 J. A. Sillero, D. Ortega, E. Muñoz Serrano, and E. Casado, J. Phys. D: Appl. Phys. 43, 185204 (2010).

13 A. Bergner, M. Westermeier, C. Ruhrmann, P. Awakowicz, and J. Mentel, J. Phys. D: Appl. Phys. 44, 505203 (2011).

14 J. Pelletier and C. Pomot, Appl. Phys. Lett. 34, 249 (1979).

${ }^{15}$ P. L. Kanitkar, C. V. Dharmadhikari, D. S. Joag, and V. N. Shukla, J. Phys. D: Appl. Phys. 9, L165 (2001).

${ }^{16}$ J. B. Taylor and I. Langmuir, Phys. Rev. 44, 423 (1933).

${ }^{17}$ R. W. Gurney, Phys. Rev. 47, 479 (1935). 
${ }^{18}$ V. Fomenko, Handbook of Thermionic Properties.

(1966).

${ }^{19}$ N. D. Lang, Phys. Rev. B 4, 4234 (1971).

${ }^{20}$ G. N. Hatsopoulos and E. P. Gyftopoulos, "Thermionic Energy Conversion”, vols. 1 (MIT Press, Cambridge, MA, 1973).

${ }^{21}$ J. J. C. Geerlings, Kwakman, and J. Los, Surf. Sci. 184, 305 (1987).

${ }^{22}$ V. Vlahos, J. H. Booske, and D. Morgan, Phys. Rev. B 81, 054207 (2010).

${ }^{23}$ P. Hohenberg and W. Kohn, Phys. Rev. 136, B864 (1964).

${ }^{24}$ D. Vanderbilt, Phys. Rev. B 41, 7892 (1990).

${ }^{25} \mathrm{https}$ ://wiki.fysik.dtu.dk/

${ }^{26}$ B. Hammer, L. B. Hansen, and J. K. Nørskov, Phys. Rev. B 59, 7413 (1999).

${ }^{27}$ W. Kohn and L. J. Sham. Phys. Rev. 140, A1133 (1965).

${ }^{28}$ N. E. Singh-Miller and N. Marzari, Phys. Rev. B 80, 235407 (2009).

${ }^{29}$ P. Soukiassian, R. Riwan, C. Guillot, J. Lecante, and Y. Borensztein, Phys. Scr. 1983, 110 (2007).

${ }^{30}$ C. Stampfl and M. Scheffler, Surf. Rev. Lett. 2, 317 (1995).

${ }^{31}$ E. Wimmer, A. J. Freeman, M. Weinert, H. Krakauer, J. R. Hiskes, and A. M. Karo, Phys. Rev. Lett. 48, 1128 (1982).

${ }^{32}$ E. P. Gyftopoulos and J. D. Levine, J. Appl. Phys. 33, 67 (1962).

${ }^{33}$ T. A. Flaim and P. D. Ownby, Surf. Sci. 32, 519 (1972).

${ }^{34}$ H. Kawano, Prog. Surf. Sci. 83 (2008) 1-165.

${ }^{35}$ K. L. Jensen, D. W. Feldman, N. A. Moody, and P. G. O'Shea, J. Appl. Phys. 99, 124905 (2006).

${ }^{36}$ C.-S. Wang, J. Appl. Phys. 48, 1477 (1977).

${ }^{37}$ G. A. Haas, A. Shih, and C. R. K. Marrian, Appl. Surf. Sci. 16, 139 (1983).

${ }^{38}$ R. T. Longo, E. A. Adler, and L. R. Falce, Tech. Dig.-Int. Electron Devices Meet. 1984, 12.2.

${ }^{39}$ A. L. Allred, J. Inorg. Nucl. Chem. 17 (3-4): 215-221 (1961).

${ }^{40}$ L. W. Swanson and R. W. Strayer, J. Chem. Phys. 48, 2421 (1968).

${ }^{41}$ Z. Sidorski, I. Pelly, and R. Gomer, J. Chem. Phys. 50, 2382 (1969).

${ }^{42}$ A. G. Fedorus and A. G. Naumovets, Surf. Sci. 21, 426 (1970).

${ }^{43}$ J. L. Desplat, C.A. Papageorpoulos, Surf. Sci. 92, 97-118 (1980).

${ }^{44}$ R. Smoluchowski, Phys. Rev. 60, 661 (1941).

${ }^{45}$ R. Cortenraad, A. W. Denier van der Gon, H. H. Brongersma, G. Gärtner, and A. Manenschijn, Applied Surf. Sci. 191, 153 (2002).

${ }^{46}$ N. Egede Christensen and B. O. Seraphin, Phys. Rev. B 4, 3321 (1971). 